\title{
Utilisation de prismes jumelés et de prismes différentiels pour améliorer la posture
}

\author{
Jonathan Shapiro, \\ B.Sc. (spécialisé), FCOptom, \\ FAAO, FEAOO \\ Optométriste, \\ Ancien conférencier principal \\ Département d'optométrie \\ Hadassah Academic College \\ Jérusalem
}

\section{Résumé}

Cet article présente des cas de changements posturaux et l'utilisation de prismes pour soulager les symptômes causés par les syndromes A et V (avec une suggestion quant à leur cause); l'asymétrie de la position de l'apophyse mastoïde; l'asymétrie faciale résultant d'un taux de croissance inégal du crâne; le relâchement de la mâchoire; le syndrome de Duane et la paralysie du $6^{\text {e }}$ nerf, ainsi que le dos voûté chez les patients âgés. Bien que les prismes jumelés et différentiels soient utilisés pour traiter et atténuer les symptômes éprouvés par les patients qui ont subi un traumatisme craniocérébral léger (TCCL), cela dépasse la portée du présent article. Les méthodes d'examen des patients et les prismes prescrits sont décrits.

\section{MOTS CLÉS :}

Prismes jumelés (yoked prisms), prismes différentiels, syndromes A et V, posture, chute, perception et localisation spatiale.

Un prisme différentiel modifie la localisation spatiale d'une image dans un œil. Les prismes jumelés sont une paire de prismes de puissance et de direction égales qui changent la localisation spatiale des deux yeux de façon égale. Les prismes différentiels sont habituellement prescrits pour atténuer les symptômes du stress de la vision binoculaire, tandis que les prismes jumelés sont utilisés pour améliorer la posture. Une prescription peut combiner les deux types de prismes. Les effets secondaires des prismes comme la distorsion, l'expansion et la compression spatiale seront abordés plus loin dans le document.

Les effets des prismes jumelés et des prismes différentiels sur la posture sont bien établis dans la littérature ${ }^{1-4}$. Kaplan ${ }^{1}$ a jeté les bases de l'utilisation des prismes jumelés verticaux dans les soins optométriques pour atténuer une variété de symptômes associés à la perception de l'espace visuel. Kaplan a mis l'accent sur l'accommodation et la convergence. Des recherches ont montré que les prismes jumelés verticaux ont des effets mineurs sur l'accommodation et la vision binoculaire, au moins pendant un port de courte durée, chez les jeunes adultes ayant une vision binoculaire normale $^{5,6}$. Cet article n'aborde pas le traitement des anomalies de la vision binoculaire, mais se concentre plutôt sur le traitement et la prévention des dommages et de l'inconfort causés par l'adaptation posturale.

Cet article poursuit le travail des auteurs précédents et s'intéresse à l'utilisation de prismes jumelés et différentiels dans la pratique courante pour modifier la posture. Les exemples de cas présentés ici décrivent l'utilisation de prismes pour diverses conditions.

Bien que les prismes jumelés aient été largement utilisés pour améliorer les capacités fonctionnelles dans les cas de traumatisme craniocérébral (TCC) et d'autres affections neurologiques, ces applications ne sont pas prises en compte dans le présent document ${ }^{3,7-10}$. Toutefois, étant donné que bon nombre des concepts présentés dans ce document sont influencés par la recherche et l'expérience acquises dans le traitement des TCC, il est pertinent d'inclure ces renseignements ici. 
La localisation égocentrique (LE) a été définie comme étant la conscience des positions spatiales des objets dans l'environnement immédiat par rapport à son corps. L'absence de cet état est appelée localisation égocentrique anormale (LEA) ${ }^{11,12}$ ou syndrome de déviation de la ligne médiane (SDLM). Le premier terme a été privilégié récemment, car on ne sait pas si la « déviation » est purement visuelle. Bien qu'elle puisse changer, par exemple, avant et après l'activité vestibulaire, elle peut très bien répondre aux prismes jumelés; il s'agit donc probablement d'un mélange de composantes visuelles et vestibulaires ${ }^{11,12}$. Padula et ses collègues ${ }^{11,12}$ ont noté que, dans des conditions normales, la ligne médiane visuelle est établie et stabilisée au début du développement du système nerveux central. Cela détermine l'alignement approprié du corps pour maintenir l'équilibre pendant le mouvement ${ }^{13}$. L'alignement postural préserve l'équilibre en intégrant l'information venant du système visuel à la base d'appui proprioceptive et à l'information qui est fournie par le système vestibulaire. La combinaison crée une ligne médiane visuelle stable.

On a proposé que l'œil dominant de la perception spatiale puisse contribuer à cet équilibre. Cette notion est étayée par les résultats de Velay et coll. ${ }^{14}$, qui ont montré que les signaux proprioceptifs provenant des deux yeux sont impliqués dans la localisation visuelle égocentrique. On a observé une dominance proprioceptive, mais de l’oil dominant.

Si l'œil dominant est endommagé, par exemple par une DMLA monoculaire ou une cataracte, la ligne médiane visuelle peut être perturbée. L'œil non dominant en perception spatiale a maintenant une bien meilleure vision que l'œil auparavant utilisé pour déterminer la localisation spatiale. Dans le présent document, cette condition s'appelle la perception spatiale déplacée. Des prismes jumelés latéraux peuvent être prescrits pour déplacer l'image perçue près de la localisation spatiale habituelle.

L'utilisation de prismes jumelés crée la base d'une nouvelle réaction visuomotrice et motrice-sensorielle qui entraîne des changements de comportement $\mathrm{t}^{1,11-13}$ Les prismes jumelés provoquent des changements de posture, car ils affectent la perception de l'espace visuel périphérique. Le système visuel peut influer sur la façon dont une personne ajuste la position de sa tête pour atteindre un équilibre confortable des muscles extraoculaires (MEO), en plus d'une bonne perception de la ligne médiane visuelle et d'une bonne localisation égocentrique.

L'utilisation de prismes jumelés ou différentiels ne traite pas la condition sous-jacente. Les prismes de l'ordonnance produisent plutôt un mécanisme compensatoire. Cela atténue le besoin de réajustement postural adopté par le patient pour réduire les symptômes d'inconfort oculaire.

Le maintien de la posture est assuré par une combinaison de nombreux facteurs. Pour maintenir l'équilibre, l'information transmise par le système visuel, le système vestibulaire et les récepteurs du système proprioceptif sensibles à la pression du cou et des pieds au sol doit être synchronisée. Le système visuel est le système dominant et peut supplanter les deux autres systèmes. On peut le démontrer en faisant marcher un sujet dans un tunnel dont les parois arborent un motif à damiers. Lorsque le motif à damier des parois du tunnel tourne, le sujet tombe dans la direction de la rotation, bien que le système vestibulaire et le système proprioceptif fonctionnent normalement ${ }^{15}$.

L'effet de la posture sur le système visuel et l'effet du système visuel sur la posture sont connectés. Nous ne savons cependant pas s'il y a un élément dominant ou si les deux éléments sont d'importance égale.

Nous pouvons supposer qu'en améliorant la posture par le réalignement et la relaxation de la tension des MEO, nous pouvons améliorer le maintien de l'espace visuel périphérique nécessaire à l'équilibre. Cela présente l'avantage supplémentaire de réduire la possibilité de dommages à long terme au cartilage rachidien et aux muscles de soutien, ce qui doit également être pris en compte.

\section{RELATION POSSIBLE ENTRE LES SYNDROMES A ET V ET LA ROTATION DE L'ORBITE}

On parle de syndromes $\mathrm{A}$ et $\mathrm{V}$ lorsque la vision binoculaire, sans tension ou avec une réduction de la tension sur les muscles extraoculaires, n'est possible qu'en dirigeant le regard vers le haut (A) ou vers le bas (V) par rapport à la position primaire. Lorsque la personne regarde dans la direction opposée à la position de confort, la vision des deux yeux a tendance à se dissocier (exophorie), ce qui rend difficile le maintien d'une vision binoculaire unique.

Dans les cas de syndrome $\mathrm{A}$, on a tendance à pencher la tête vers le bas pour élever les yeux au sommet du A, position dans laquelle le maintien de l'équilibre des muscles des yeux pour une vision binoculaire est le plus confortable. Cette position de tête est inversée dans les cas de syndrome V. Il a été proposé qu'une autre raison de pencher la tête vers le bas et d'élever les yeux dans l'orbite est que cette position pourrait permettre de créer une forme d'amortissement des oscillations micronystagmiques causées par le stress sur les MEO en position primaire. 
Quaid et Hamilton-Wright ${ }^{16}$, ont noté en comparant le test de Hess au test des 3 étapes de Parks que, dans le cas d'une ésodéviation, il y aura un modèle différent, et les mouvements seront inversés.

Les anomalies de vision binoculaire rencontrées avec les syndromes A et V résultant de trophies (généralement $15^{\Delta}$ différences entre les deux positions de regard) ne sont pas prises en compte ici. Celles-ci sont normalement causées par des problèmes d'alimentation nerveuse, d'insertion musculaire mal positionnée ou de sous-action musculaire.

La cause des syndromes A et V non pathologiques n’a pas été entièrement déterminée. Urrets-Zavalía et ses collaborateurs ${ }^{1718}$ ont présenté un concept intéressant. Travaillant en Argentine dans les années 1950, ils ont noté que la population indigène pure (désignée comme mongolö̈de) avait des fentes palpébrales avec un canthus externe plus haut, tandis que la population non indigène (Européenne pure, appelée antimongoloïde) avait tendance à avoir des fentes palpébrales droites ou avec un canthus externe plus bas. Ils ont proposé que la position de la rotation orbitale avait une influence sur l'emplacement de l'insertion des MEO. Il est suggéré ici que cela pourrait être la cause des syndromes A et V. Urrets-Zavalía et ses collaborateurs considéraient que la position de rotation des orbites est influencée par le développement relatif de l'os zygomatique (pommette). Si l'os zygomatique se développe comme prévu, les orbites des yeux sont de niveau et les points d'insertion des droits latéral et médial se trouvent sur le même plan. Le développement hypoplasique de l'os zygomatique entraînera une rotation en dehors du globe oculaire, tandis que le développement hyperplasique créera rotation du globe en dedans. Cela sera noté par l'apparence des fentes palpébrales. Dans leurs mots, «(i)il apparaît donc que les troubles susmentionnés des muscles oculaires sont en quelque sorte liés aux troubles accompagnant l'échafaudage facial. » (Traduction libre)

Lorsque les fentes palpébrales semblent horizontales, en supposant que les orbites suivent la structure de la position palpébrale, les mouvements de vergence maintiennent leur position relative et se font en douceur. Il n'y a pas de tension cyclique sur les MEO pendant la vergence ou la convergence. La tête garde une posture droite.

Lorsqu'il y a développement hypoplasique de l'os zygomatique, le développement de la rotation orbitale n'est pas terminé. Le droit médial se trouve sur un plan plus élevé que le droit latéral. Cela entraîne un syndrome V et le menton est levé.

Lorsqu'il y a développement hyperplasique de l'os zygomatique, il y a une structure orbitale en dedans. Le droit latéral est placé plus haut que le droit médial, un syndrome A se produit et le menton est abaissé.

Un développement asymétrique de l'os zygomatique fait en sorte qu'une des orbites est « droite » alors que l'autre orbite est en dedans ou en dehors. Il y aura également une asymétrie faciale verticale. Soit dit en passant, si l'orbite ayant un canthus externe plus haut est l'œil dominant, la personne aura tendance à incliner sa tête lorsqu'elle suit des yeux une cible en mouvement pour aligner les droits médial et latéral (Fig. 1).

Figure 1 : Position relative des orbites. (a) Ouverture palpébrale droite. (b) Canthus externe plus haut, bilatéral. (c) Canthus externe plus bas, bilatéral. Asymétrie : (d) Structure orbitale de droite en dehors, orbite gauche droite. (e) Structure orbitale de droite en dehors, orbite gauche droite et élevée.

(a)

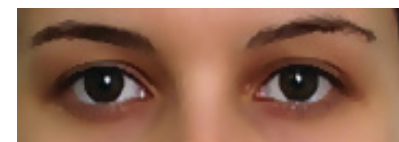

(d)

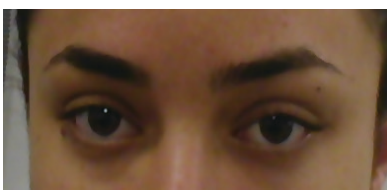

(b)

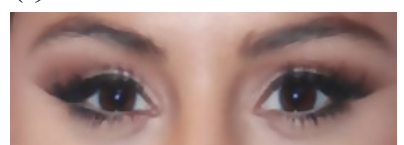

(e) (c)

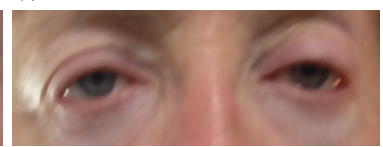

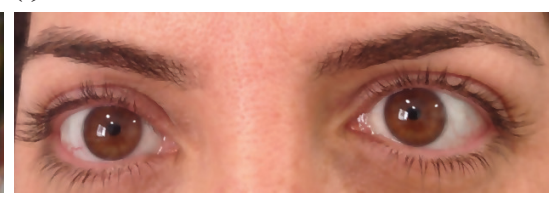

Le menton levé ou rentré affecte la posture. Des adaptations sont faites dans la colonne vertébrale pour maintenir le centre de gravité. 
MÉTHOdE DE SURVEILLANCE DE LA POSITION RELATIVE DES YEUX PENDANT LES MOUVEMENTS DE VERGENCE Le matériel servant à surveiller la position relative des yeux pendant les mouvements de vergence n'est généralement pas disponible dans le cadre du travail clinique. Le système suivant a été conçu pour analyser l'effet des déséquilibres verticaux et guider la prescription des prismes jumelés.

Il est possible d'établir les variations de la position verticale relative d'un objet perçu en mesurant soigneusement l'équilibre vertical pendant que la tête se déplace lentement à gauche et à droite alors que le regard est fixé sur une cible centrale. On examine la position relative en maintenant la tête en position primaire, en répétant avec le menton rentré, et à nouveau en relevant le menton.

Figure 2 : (a) Cibles utilisées pour déterminer la position relative des yeux en poursuite oculaire. Les lignes de nonius sont orientées horizontalement et verticalement. (b) Parallel-Testing Infinity Balance (PTIB). La cible entière est vue par les deux yeux simultanément, à l'exception des carrés et de la ligne rouge qui y est attachée, vus par chaque œil séparément, qui sont ombrés pour explication.

(a)

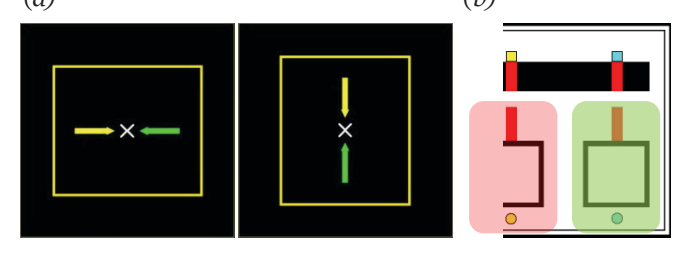

L'examen utilise différentes cibles (Fig. 2). La première cible a deux lignes placées le long de l'horizontale, polarisées pour chaque œil. Les couleurs choisies pour les flèches sont proches l'une de l'autre dans le spectre afin d'éviter le déplacement spatial de l'aberration chromatique transversale (ACT). L'utilisation d'un carré jaune permet le maintien de la vision périphérique. Les positions des lignes par rapport au X, et entre elles, sont comparées lorsque la tête se déplace latéralement tout en gardant le regard fixé sur le X. L'examinateur tient la tête du patient en position primaire pour empêcher le basculement de la tête. En tenant la tête pendant l'examen, l'examinateur peut contrôler la vitesse du mouvement. La tête se déplace lentement à gauche et à droite. Si aucune variation de la position verticale des images n'est constatée, on peut en déduire que la position du droit latéral et du droit médial est de niveau. Une différence importante dans la hauteur verticale relative des deux yeux, à la limite des mouvements de vergence, indique un déséquilibre. Si les muscles droit latéral et droit médial ne sont pas alignés, une ligne monte ou descend lorsque la tête se déplace d'un côté. En se déplaçant dans la direction opposée, la ligne opposée se déplace. Cette action est répétée lorsque le menton est rentré et relevé. S'il y a une réduction du mouvement vertical pendant les mouvements de vergence en comparant la position primaire au moment où le menton est rentré ou relevé, des prismes jumelés verticaux sont introduits. La puissance des prismes jumelés est déterminée par la déviation prismatique nécessaire pour réduire le mouvement vertical au minimum, lorsque la tête est maintenue en position primaire. Après l'introduction des prismes jumelés, le test est répété avec en positionnant la ligne de nonius verticalement, pour déterminer s'il y a une perturbation de l'équilibre horizontal.

Le cadre d'essai doit être placé avec soin pour éviter d'avoir à utiliser des prismes jumelés dans des prescriptions fortes. Si la prescription est faible, il est préférable d'effectuer l'examen sans lunettes. S'il y a des cylindres obliques dans l'ordonnance, l'examen est compromis en raison de la dislocation spatiale causée par les mouvements latéraux.

La troisième cible, le Parallel-Testing Infinity Balance (PTIB), ${ }^{19,20}$, a de multiples utilisations pour examiner la relation de l'espace visuel, vu par chaque œil, avec l'espace visuel binoculaire.

La barre noire supérieure, avec deux lignes rouges, est vue de façon binoculaire. Les carrés, avec des lignes rouges attachées, sont vus séparément par chaque œil. Dans le cadre de ce test, le tableau PTIB montrera le désalignement vertical et horizontal des images perçues par chaque œil pendant le mouvement. Cela est perçu par le patient comme des changements dans l'alignement vertical des deux carrés et/ou dans la position horizontale relative des lignes rouges sur les carrés par rapport aux lignes rouges dans la barre noire. L'examen relèvera également toute cyclorotation ou variation de la taille de l'image. Il mettra aussi en évidence l'implication possible des muscles obliques.

La tête est maintenue en position primaire. Au besoin, des prismes différentiels sont utilisés pour équilibrer les déséquilibres vertical et horizontal avant le déplacement de la tête. Une fois la position des carrés en équilibre, la tête est déplacée latéralement et verticalement, comme il a été décrit précédemment. Le patient indique si les carrés demeurent au même niveau, s'il y a cyclotorsion par rapport à la forme originale, s'ils changent de taille ou si la ligne rouge sur les carrés se 
dissocie de la ligne rouge de la barre noire au-dessus. L'information permet à la personne qui fait l'examen de déduire les actions des MEO. Il est moins important de savoir s'il y a un muscle en particulier qui travaille moins ou davantage que de déterminer la relation entre les muscles jumelés pendant la poursuite oculaire. Une cyclotorsion, telle qu'elle est visualisée sur les carrés du PTIB, peut être modifiée lorsque la tête est maintenue dans une position précise.

La position des orbites peut également être influencée par le sous-développement ou le développement excessif de la projection nasale, ce qui provoque un déplacement ver l'intérieur ou l'extérieur des orbites (Fig. 3).

Figure 3 : (a) sur-développement, (b) sous-développement et (c) développement asymétrique de la Figure 4.

(a)

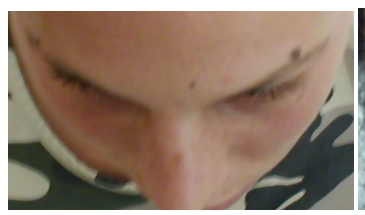

(b)

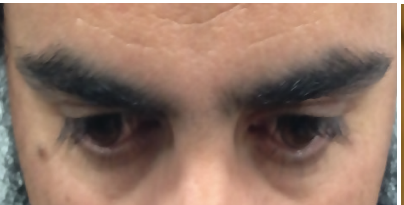

(b)

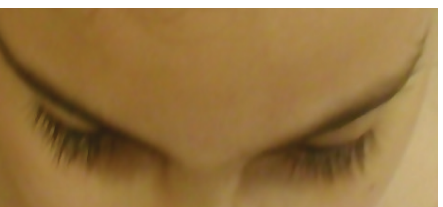

\section{EFFETS DES SYNDROMES A ET V SUR LA POSTURE}

La gravité des déséquilibres de la vergence et de l'accommodation, la souplesse du système avec modification de la position de la tête et l'introduction d'un prisme vertical ont fait l'objet de débats. Quaid et Simpson ${ }^{21}$ ont montré une corrélation avec l'efficacité de lecture à l'aide du suivi infrarouge. Dans les cas d'inclinaison excessive de la tête vers l'avant ou vers l'arrière, une formation était nécessaire pour améliorer la flexibilité accommodative. La relaxation du stress sur les muscles du cou réduit le déséquilibre et, avec le temps, améliore le confort d'une personne lorsqu'elle lit (Fig. 4). Le traitement de la flexibilité accommodative par la formation peut être suffisant pour atténuer les problèmes et peut remplacer les prismes jumelés ou être utilisé en conjonction avec eux. D'autres recherches ont conclu que l'effet des prismes verticaux sur la phorie horizontale n'a aucune conséquence, bien que les syndromes A et V n’aient pas été pris en compte dans ce rappor ${ }^{6}$.

Un patient présentant un syndrome A peut baisser sa tête en rentrant son menton, ce qui pousse l'œil dans l'orbite supérieure. Lorsque le menton s'enfonce, le centre de gravité du corps est déplacé vers l'avant. Pour compenser ce changement, le dos est courbé vers l'intérieur. C'est l'inverse qui se produit dans le cas du syndrome du V. En incorporant des prismes jumelés dans l'ordonnance, la position de la tête est adaptée, ce qui atténue le stress sur la posture et facilite la poursuite oculaire.

Figure 4 : (a) Syndrome A sans et avec correction au moyen de prismes jumelés à base orientée vers le bas (BB) (b) Dos courbé traité à l'aide de prismes jumelés $B B$.

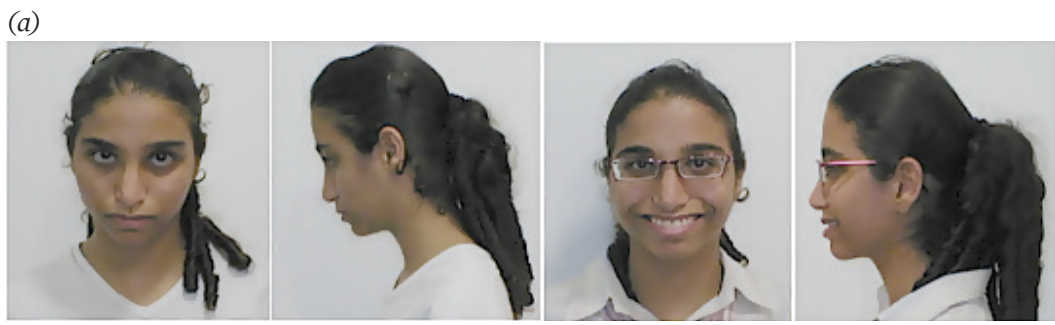

(b)

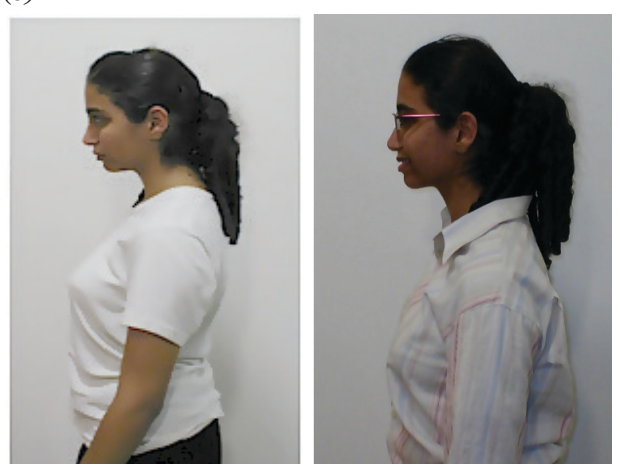




\section{ASYMÉTRIE DE LA POSITION DE L’APOPHYSE MASTOÏDE}

L'asymétrie verticale du visage (AVV), soit en raison de la croissance génétique différentielle du crâne, soit en raison du raccourcissement des muscles sterno-cléido-mastoïdiens (SCM), cause une déformation du crâne et peut également influencer l'inclinaison de la tête. ${ }^{22,23}$ Le tendon supérieur du SCM est ancré dans l'apophyse mastoïde. Le SCM joue un rôle important dans le contrôle de la position posturale de la tête.

Les muscles SCM, trapèze et sous-occipital commencent à fonctionner environ 3 mois après la naissance, soit environ un mois avant le développement de la convergence, de la divergence et de la fusion. S'il y a une inclinaison dans la position du crâne en raison d'une asymétrie ou d'un déséquilibre de ces muscles, il est possible que les vergences ne se développent pas à leur plein potentiel. L'emplacement relatif des yeux peut induire une cyclorotation. Major et ses collègues ${ }^{24}$ ont noté un taux élevé de troubles de la coordination oculomotrice chez les enfants qui avaient été confinés à un fauteuil roulant dès leur jeune âge.

La position des apophyses mastoïdes de droite et de gauche peut ne pas être symétrique. Une asymétrie antérieure-postérieure provoque une tension sur l'axe des y de la tête. À la figure 5, l'apophyse mastoïde de droite est située plus loin derrière l'apophyse mastoïde de gauche. La tête est tournée sur l'axe des y jusqu'à l'épaule droite. Le patient dispose de divers mécanismes compensatoires pour maintenir la position du corps et de la tête. Cela dépendra des mouvements requis. La marche exige une position symétrique des hanches et des épaules. Pour ce faire, il y aura un mouvement de vergence vers la gauche. En lecture et à l'ordinateur, le patient gardera la tête droite et utilisera plutôt un mouvement sur l'axe des y des épaules et des hanches. La marche monotone sur un tapis roulant en regardant un écran de télévision placé directement à l'avant peut perturber les muscles de l'épaule et du bas du dos. Pour garder la tête tournée, l'épaule droite se déplace vers l'avant, ce qui est compensé par une torsion de la colonne inférieure dans la direction opposée. La tension du muscle SCM nécessaire pour garder la tête et la colonne droites peut causer des douleurs au cou. Les patients qui ont cette structure ont des préférences pour certaines positions assises lorsqu'ils regardent la télévision, sont au théâtre ou dans une salle de cours, quand ils placent l'ordinateur sur le bureau, etc. S'ils sont assis dans une position opposée, ils se tordent, ce qui entraîne un stress musculaire. Les prismes jumelés horizontaux peuvent être utilisés si la position de la tête tournée est maintenue et la vergence constante perturbe la poursuite oculaire normale. Il convient de noter que pour les multifocales, la mesure de la D.I. de chaque œil doit être effectuée en position naturelle, et non avec la tête droite.

Figure 5: Asymétrie antérieure-postérieure de la position de l'apophyse mastoïde. La tête est tournée vers la droite.

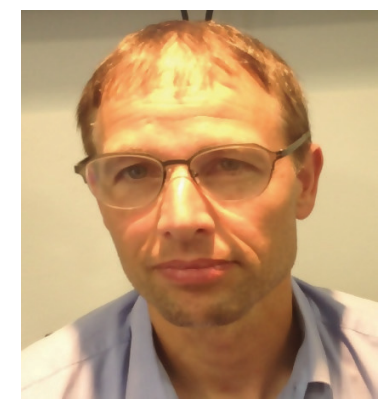

\section{RACCOURCISSEMENT DU MUSCLE SCM}

Une autre forme d'asymétrie est causée par la malformation du crâne. Le positionnement du crâne dans une position exiguë dans l'utérus, surtout si la tête était placée en haut, peut être une des causes du torticolis congénital. La déformation du crâne se produit habituellement du côté droit, bien qu'on la trouve parfois du côté gauche. Étant donné que la déformation se produit habituellement du côté droit, on suppose que la position latérale déplacée du foie, qui appuie sur l'utérus, peut être un facteur contributif lorsque la tête est positionnée vers le haut. Il est possible qu'un seul de deux jumeaux identiques présente la déformation, ce qui suggère qu'il n’y a pas d'implication génétique (Fig. 6).

Le SCM est plus court, plus épais et moins flexible d'un côté, habituellement du côté droit. Il est difficile de redresser la tête. Le déplacement de la tête sur l'axe des y est libre dans une direction, mais limité dans la direction opposée. Le patient peut ajuster sa posture pour atténuer le stress de ce côté. La différence dans la hauteur verticale des yeux est plus marquée que dans la première forme d'asymétrie mentionnée ci-dessus. Les épaules sont plus arrondies et plus épaisses que la normale et sont asymétriques. La colonne vertébrale montre une courbure au cou (Fig. 7). Les ouvertures palpébrales sont souvent réduites. L’inclinaison de la tête entraîne une cyclorotation des yeux. Selon la gravité de 
l'inclinaison de la tête, la fonction binoculaire peut être mauvaise, souvent avec suppression dans un œil. Si la tête est redressée, la fonction binoculaire s'améliore, mais le stress sur le muscle SCM plus épais raccourci cause de la douleur.

Le problème est traité par physiothérapie, notamment l'étirement du muscle et des tendons touchés. Un prisme différentiel, combiné à la physiothérapie, est utilisé pour égaliser la position de l'image perçue dans chaque œil.

Figure 6: (a) Jumeaux mâles identiques. Le frère de droite a un raccourcissement asymétrique du muscle SCM droit. (b) Le côté du visage qui a un SCM plus court et plus épais empêche la croissance normale du crâne. Ce côté est plus petit et plus étroit que le côté non touché. L'oreille est située plus bas et la tête s'incline dans cette direction. Habituellement, l'anomalie se trouve du côté droit (gauche), mais elle peut se produire du côté gauche (droite).

(a)

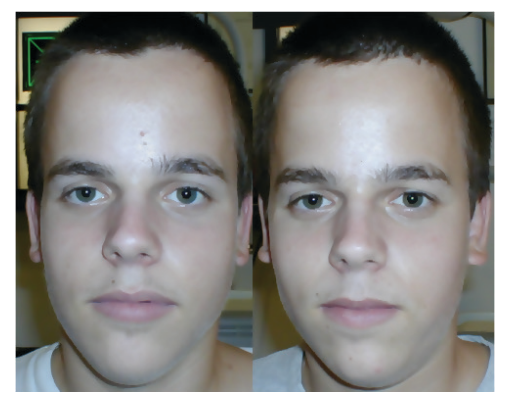

(b)

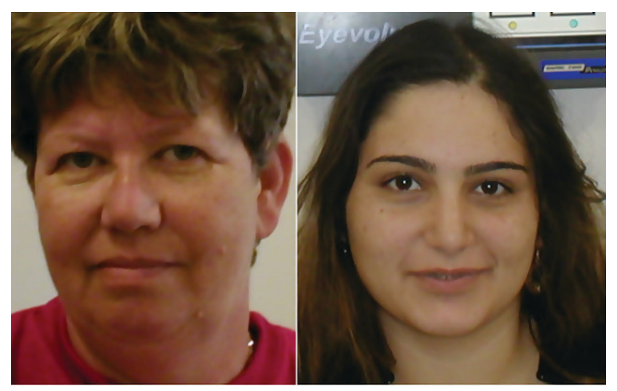

Figure 7: (a) Raccourcissement du muscle SCM du côté droit. Les yeux ne sont pas positionnés au même niveau. Il y a une légère cyclorotation des yeux. Les ouvertures palpébrales sont souvent réduites. (b) Les épaules se développent avec une structure différente. (c) Il peut y avoir un léger déplacement de la colonne cervicale.

(a)

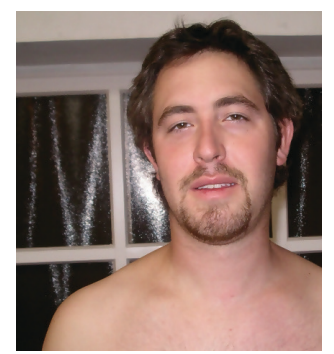

(b)

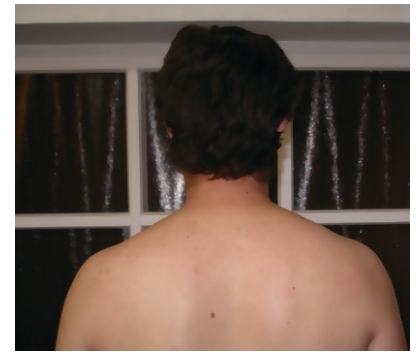

(c)

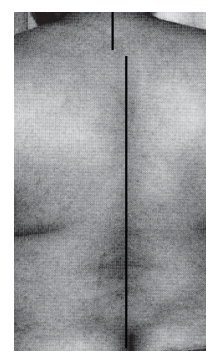

Dans la figure 8, le patient a été traité par l'introduction initiale d'un prisme de $1^{\Delta}$ prismatique base est orientée vers le bas (BB) OD, dont la puissance devait être augmentée au besoin en cours de traitement. Il est important de continuer à étirer le muscle SCM droit avec une physiothérapie agressive. Cette utilisation du prisme combinée à la physiothérapie a redressé la tête et réduit la cyclorotation. La poursuite oculaire s'est améliorée. Sans physiothérapie, l'introduction d'un prisme pour allonger le SCM droit entraîne une petite courbure compensatrice au niveau des épaules et du bas du dos et nuit au patient.

Figure 8: (a) Le raccourcissement du muscle SCM du côté droit entraîne un basculement de la tête vers l'épaule droite. Les yeux ne sont pas positionnés au même niveau. Il y a cyclorotation. La capacité de poursuite oculaire et la stéréopsie sont réduites. (b) L'utilisation de la physiothérapie et de prismes verticaux différentiels a redressé la posture de la tête.

(a)

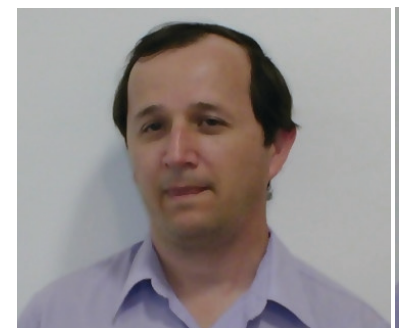

(b)

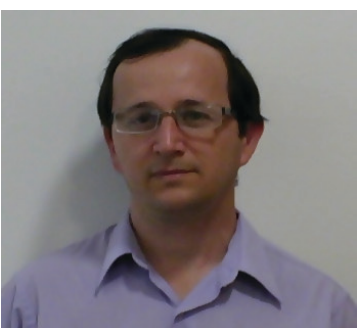


La figure 9 montre une forme simple de physiothérapie qui peut être effectuée à domicile. Cette thérapie dure quelques minutes par jour jusqu'à ce que la flexibilité requise soit atteinte. La tête du sujet est tenue contre la poitrine de la personne qui effectue l'étirement pour protéger la colonne cervicale. La personne qui effectue l'étirement utilise un bras comme contre-tension tandis que le deuxième bras applique une tension. Lorsqu'on atteint la limite de la position de confort, on applique une légère tension supplémentaire pour allonger les ligaments. L'étirement est fait dans les axes $\mathrm{x}$ et y. Il est bon d'effectuer l'étirement dans les deux sens, bien qu'une direction soit habituellement flexible.

Figure 9: Étirements pour allonger les ligaments du sterno-cléido-mastö̈dien. En équilibrant la tension des deux muscles SCM, la tête peut être maintenue droite et la colonne vertébrale alignée.

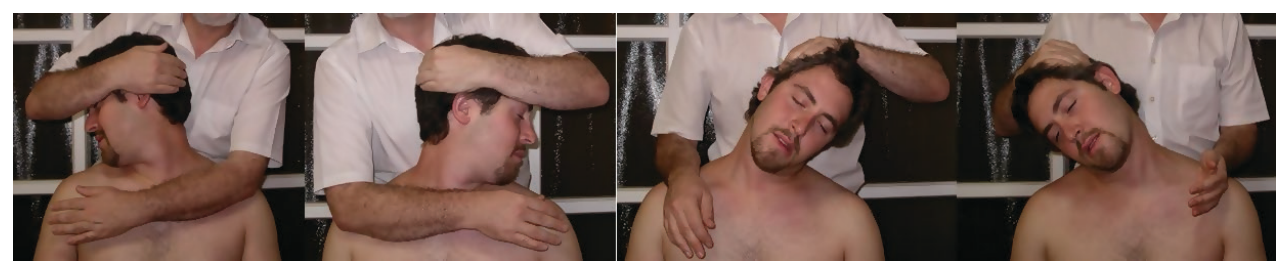

La patiente de la fig. 10 souffrait d'épiphora sur la joue droite. La patiente présentait un développement hypoplasique de l'os zygomatique droit et un raccourcissement du muscle SCM droit. La tête était penchée vers la droite, le petit canthus étant considérablement plus bas que le grand canthus. Cela a inversé l'écoulement naturel des larmes, et l'écoulement s'est éloigné du canal lacrymal. Cela avait pour résultat une épiphora sur la joue droite. L'introduction d'un prisme de $1,5^{\Delta}$ prismatique BB OD a permis de redresser la posture de la tête. La direction de l'écoulement lacrymal a été inversée et l'épiphora a cessé immédiatement. La patiente a ressenti de la tension sur le SCM droit, qui a été traité au moyen de physiothérapie et d'étirement des muscles du cou.

Figure 10: Utilisation d'un prisme différentiel vertical pour atténuer le larmoiement.

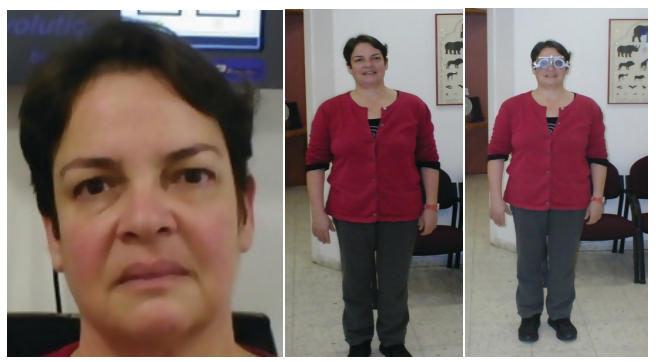

APOPHYSE MASTOÏdE DÉPLACÉE VERTICALEMENT, EN RAISON DE LA DIFFÉRENCE DE CROISSANCE GÉNÉTIQUE, SANS IMPLICATION DU SCM

Les patients peuvent avoir des muscles SCM tout aussi développés, sans le raccourcissement et l'épaississement que l'on observe avec le torticolis, mais la position de l'apophyse mastoïde est asymétrique. La tête a un mouvement libre et égal dans toutes les directions, ce qui n'est pas le cas avec des torticolis. Lorsque l'apophyse mastoïde est placée plus haut d'un côté que de l'autre, la tête tend à égaliser la tension du SCM en s'inclinant. Lorsque cela est combiné à l'asymétrie de développement du crâne antérieur-postérieur, l'apophyse mastoïde étant plus à l'arrière d'un côté du crâne, les épaules ont tendance à se courber vers l'intérieur. On a tendance à se recroqueviller. Cela réduit la profondeur de la respiration, car les poumons ne peuvent se dilater complètement. Il y a une cyclorotation des yeux, ce qui crée des problèmes de poursuite oculaire. L'introduction de prismes jumelés BB combinés à un prisme différentiel BB dans l'œil droit améliore la posture (Fig. 11).

Le patient à la figure 12 présentait des problèmes d'apprentissage et de comportement social. Son incapacité à lire facilement, son manque de progrès en matière d'apprentissage, sa maladresse et sa timidité naturelle ont rendu ses interactions sociales et son estime de soi problématiques à l'école. Il avait commencé à manger comme moyen de se réconforter et était devenu en surpoids. 
Il présentait une combinaison d'asymétrie d'apophyse mastoïde verticale et antérieure-postérieure, combinée à un syndrome A. Il avait une très légère amétropie.

En introduisant un prisme de $5^{\Delta}$ prismatiques BB OD et un prisme de $41 / 4^{\Delta}$ prismatiques $\mathrm{BB}$ OS, on a pu réduire l'inclinaison de la tête et redresser la posture. Ses capacités scolaires, sa maladresse et son acceptation sociale se sont nettement améliorées. Comme on peut le constater en regardant sa posture et son poids un an plus tard, son besoin de manger pour se réconforter a grandement diminué. Il faudra très peu de physiothérapie, car les muscles SCM ne sont pas contractés. Toutefois, il y a eu des répercussions. La dernière image montre qu'il y a eu une augmentation de la flexion de la colonne vertébrale au cou et au milieu du tronc comparativement à l'année précédente. Cela fera l'objet d'un suivi.

Figure 11: . (a) Positionnement asymétrique vertical et antérieur postérieur de l'apophyse mastö̈de. Le muscle SCM droit tire la tête vers l'épaule droite, et les deux épaules se courbent vers l'intérieur. (b) L’utilisation de prismes jumelés et différentiels permet de réaligner la tête et les épaules.

(a)

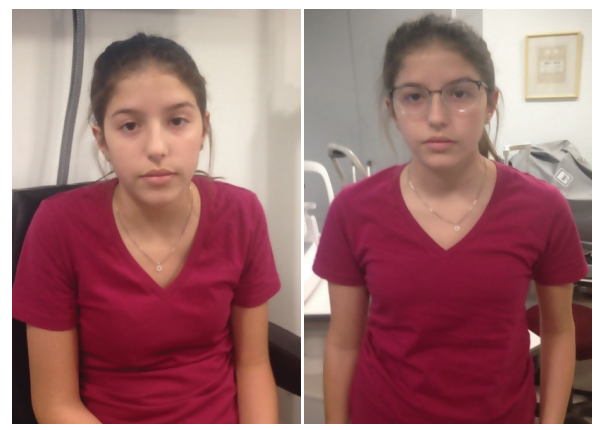

Figure 12: Changements de posture sur un an. La puissance des prismes prescrits était de $5^{\Delta} B B O D$ et de $41 / 4^{\triangle} B B O S$.
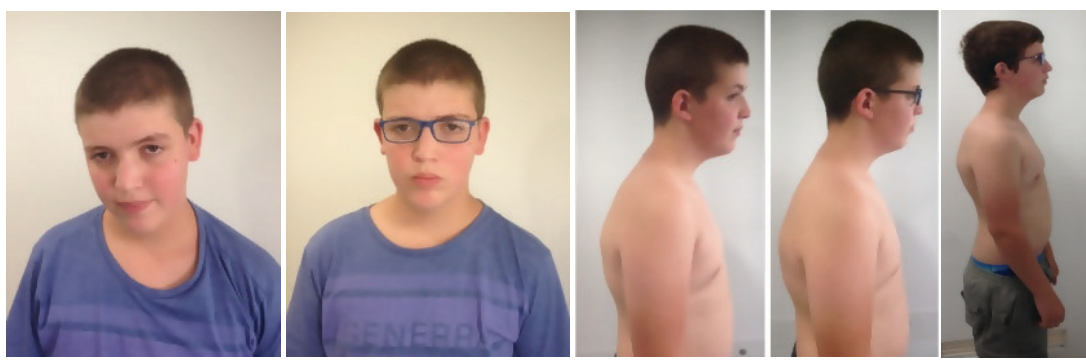

ASYMÉTRIE FACIALE ATTRIBUABLE À UNE CROISSANCE INÉGALE

Le développement asymétrique du visage peut faire en sorte qu'un œil soit placé plus haut que l'autre, sans atteinte de l'apophyse mastoïde ou du SCM.

Dans la plupart des cas, l'œil gauche est plus haut que l'œil droit, bien que l'on rencontre aussi l'inverse. Si l'activité du patient implique un mouvement du corps, sans un regard concentré, le maintien de l'équilibre est primordial et la tête reste droite. Lorsque le patient a besoin d'un regard concentré, plutôt que de voir, la tête s'incline pour égaliser la hauteur des yeux (Fig. 13). Lorsque la tête s'incline, il y a une tendance à créer une flexion latérale en « $\mathrm{S}$ » dans la posture pour équilibrer le centre de gravité22,23. C'est ce que nous constatons à la figure 14. Cela peut aussi causer une légère cyclorotation des yeux, ce qui peut avoir une incidence sur la position optimale du droit médial et du droit latéral et compromettre la poursuite oculaire. L'utilisation d'un petit prisme vertical, $1 / 4^{\Delta,} 1 / 2^{\Delta}$, repositionne l'image rétinienne, et la tête se redresse. Cela réaligne la colonne vertébrale, réduit le stress sur les muscles posturaux et le cartilage de la colonne vertébrale et améliore la poursuite oculaire. Il est suggéré que cette adaptation est due à la très lente différence de croissance dans la hauteur des yeux. Chez l'enfant, la différence de hauteur est très faible. Un modèle d'intégration binoculaire est créé dans le cortex cérébral. À mesure que le crâne croît, la position verticale relative des yeux augmente lentement, et la position de la tête change pour maintenir ce modèle original. 
Figure 13: Asymétrie faciale due à la croissance, sans implication de l'apophyse mastoïde ou du SCM. Pendant la "vision » normale, la tête demeure verticale (image de gauche pour chaque sujet). En « regardant », c.-à-d. en observant de façon concentrée, la tête bascule pour mettre les yeux au niveau (image de droite pour chaque sujet).
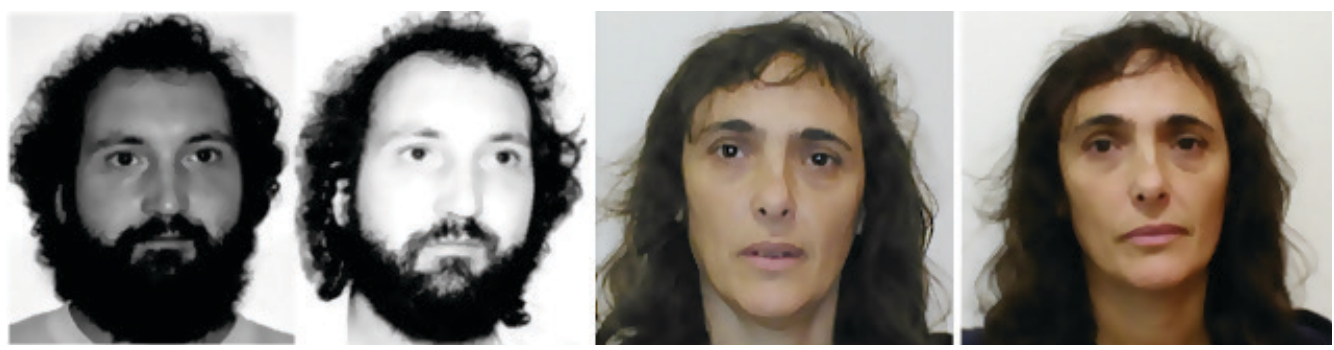

Figure 14: Pour maintenir l'équilibre, la colonne s'adapte et un coude en « $S$ » se forme.

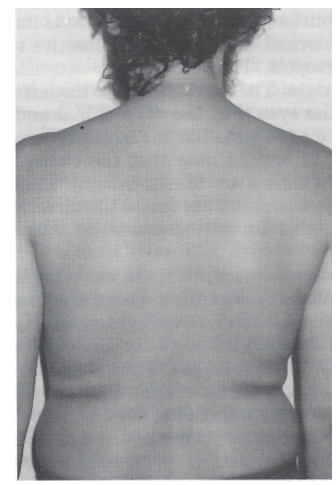

\section{SYNDROME DE DUANE ET PARALYSIE RECTOLATÉRALE}

La littérature mentionne l'utilisation de prismes pour traiter l'inclinaison horizontale de la tête, comme dans le cas du syndrome de Duane, mais ne précise pas si les prismes prescrits sont différentiels ou jumelés ${ }^{25}$.

Les prismes jumelés peuvent être utilisés dans le syndrome de Duane et la paralysie du sixième nerf crânien, quand le point nul de la vision binoculaire se produit lorsque la tête est tournée de côté. Cette rotation de la tête, est un désagrément esthétique et, lors de certaines actions monotones comme l'utilisation de l'ordinateur ou de la télévision, ainsi que la conduite, peut entraîner une torsion de l'axe des y de la colonne vertébrale et un stress sur les muscles du cou.

Figure 15: Le syndrome de Duane et la paralysie du sixième nerf. Utilisation de prismes jumelés horizontaux pour réduire les mouvements rotatoires de la tête.
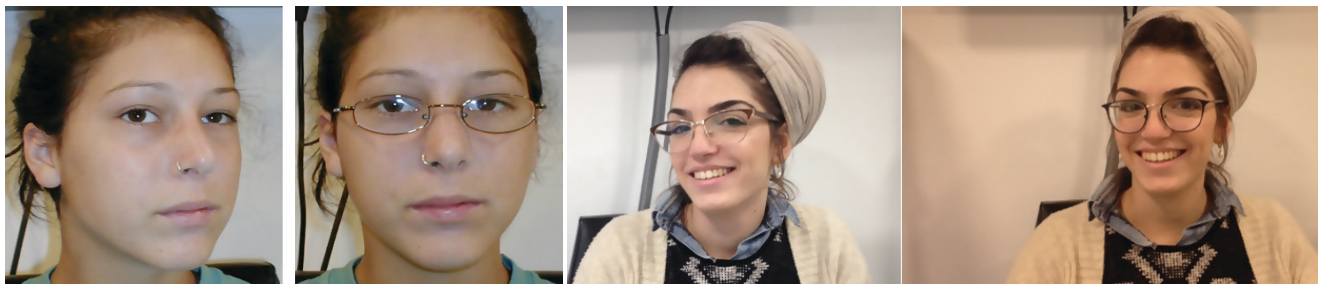

Les prismes horizontaux sont utilisés pour positionner la tête le plus droit possible. Les prismes ajoutent du poids et de l'épaisseur, ainsi qu'une réduction de l'acuité visuelle. Un compromis peut être nécessaire. Dans la figure 15, la posture de la patiente de gauche, atteinte du syndrome de Duane, est entièrement corrigée. Elle a des besoins de réfraction mineurs et ses lunettes n'ont besoin que de prismes. 
L'autre patiente, qui souffrait d'une paralysie nerveuse du sixième nerf crânien, avait besoin de prismes jumelés horizontaux droits de $12^{\Delta}$ combinés à un prisme vertical pour corriger complètement la posture. Bien que la position de la tête ait été corrigée, la patiente a rejeté la première paire de lunettes produites, avec prismes jumelés de $12^{\Delta}$ parce que la distorsion optique, le poids et l'apparence esthétique n'étaient pas acceptables. La deuxième paire $\left(8^{\Delta}\right.$ jumelés) a également été rejetée parce qu'il y avait des distorsions visuelles inacceptables. La prescription finale (acceptable) était pour des prismes jumelés droits de $6^{\Delta}$. Avec la prescription finale, la fonctionnalité dans les activités de la vie quotidienne s'est améliorée, et les maux de cou et de dos ont été réduits, mais sans disparaître complètement.

La méthode utilisée pour déterminer la force de prisme requise comprenait une corde de Brock (Fig. 16). La patiente trouve le point nul sans porter de prismes. Les prismes jumelés sont introduits tout en maintenant le point nul, jusqu’à ce que la patiente sente que le corps est aligné. Les prismes verticaux peuvent être inclus si la patiente signale que les deux lignes perçues de la corde de Brock ne sont pas de la même hauteur.

Figure 16: Utilisation d'une corde de Brock pour déterminer le point nul et la puissance de prismes jumelés requise.
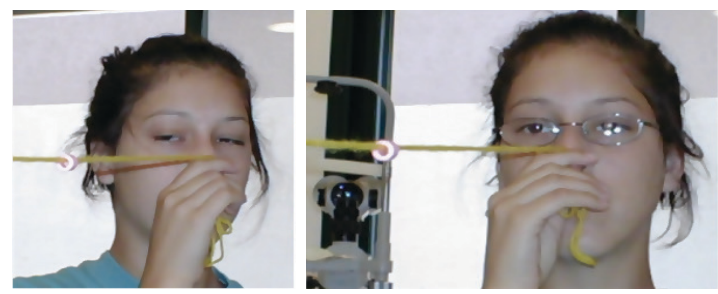

On peut aussi prescrire des prismes jumelés pour aider le patient à garder la nourriture dans sa bouche et atténuer la sécheresse de la bouche dans les cas de mâchoire tombante ${ }^{26}$. Les patients qui ont des verres à indice élevé ou une monture qui ne maintient pas sa position et glisse vers le bas regarderont à travers des lentilles qui créent un prisme BB. Cela fait en sorte que l'image apparaît plus haut. Le patient lèvera la tête et peut-être les yeux. La mâchoire a tendance à tomber davantage et il arrive que la nourriture tombe hors de la bouche pendant qu'on mange. La sécheresse de la bouche peut également s'ensuivre. En utilisant des prismes jumelés dont la base est orientée vers le haut (BH) dans les lentilles et en choisissant la monture avec soins, il est possible de maintenir la position de la tête et de réduire la perte de nourriture.

L’utilisation de prismes jumelés et différentiels pour ajuster l'espace visuel et la localisation spatiale est indépendante des besoins visuels du patient et ne doit pas être confondue avec un traitement orthoptique.

CHANGEMENTS POSTURAUX DUS À L’ÂGE

Les blessures dues aux chutes représentent la principale cause de visites aux services d'urgence des hôpitaux chez les gens âgés ${ }^{27,28}$. Le lien entre les chutes chez les personnes âgées et les causes liées à la vision est bien établi29,30.

La vision fournit des données importantes pour le contrôle de la posture, ainsi que des renseignements sur la taille et la position des dangers et des obstacles. La plupart des verres multifocaux, et certains bifocaux, ont un prisme aminci (prismes jumelés) incorporé dans leur conception, pour des raisons esthétiques et pour réduire le poids ${ }^{2,31}$. Il a été démontré que le réflexe vestibulo-oculaire (RVO) est perturbé par l'utilisation de verres multi-focaux ${ }^{3}$. L'adaptation est plus facile chez les jeunes patients, mais peut être problématique chez les porteurs plus âgés, qui ont un moins bon tonus musculaire. La prescription de lentilles unifocales, par opposition à des lentilles multifocales, a été recommandée ${ }^{29,32} \mathrm{Ce}$ document porte principalement sur la proprioception corporelle.

La perte de la force corporelle, de la masse musculaire et du tonus au fur et à mesure que le corps vieillit entraîne une courbure (Fig. 17). La tête se déplace vers l'avant et tombe. Cela déplace le centre de gravité vers l'avant. Lorsque la tête tombe, il peut être difficile de garder les yeux levés. Cela peut entrainer une perte dans le champ visuel supérieur, et des objets placés plus loin peuvent ne pas être vus. Cela réduit la capacité d'éviter les obstacles. La personne a tendance à se pencher vers l'avant et peut devoir compter sur un bâton de marche pour stabiliser le corps. Les personnes âgées ont tendance à traîner les pieds lorsqu'elles marchent, car le fait de faire un pas vers l'avant déplace le centre de gravité trop loin vers l'avant, et il y a un sentiment d'insécurité. C'est particulièrement difficile lorsqu'on se déplace dans les escaliers et sur les trottoirs. 
Figure 17: (a) Une inclinaison de la tête causée par une perte de force musculaire chez un homme âgé. Le centre de gravité est déplacé vers l'avant. Le patient traîne les pieds et ne se sent pas en sécurité. (b) L'utilisation de prismes jumelés BB déplace l'image vers le haut. La tête est relevée. Le centre de gravité est mieux aligné. La marche s'améliore.

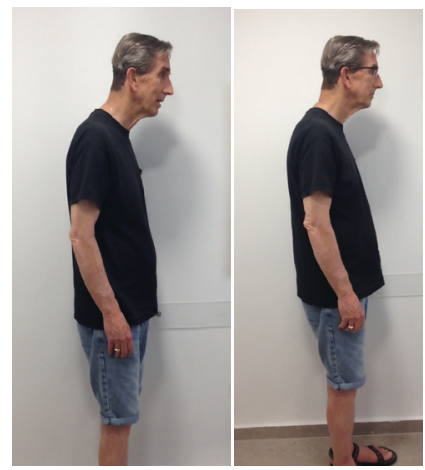

Avec l'ajout de prismes jumelés BB, le patient a tendance à relever sa tête. Le centre de gravité est déplacé vers l'arrière. Le patient compense en modifiant la position de ses genoux et de son dos. L'amélioration de la stabilité et des mouvements est le résultat d'une combinaison de facteurs. Il y a une sensibilisation périphérique accrue. Les MEO fonctionnent dans une position qui offre une plus grande marge de mouvement. L’espace périphérique perçu augmentera. Cette augmentation du signal périphérique incitera également à relever la tête. Cet effet a été noté dans le test Van Orden Star (VO Star) ${ }^{3}$.

Lorsque les sujets commencent avec des prismes jumelés, ils traînent moins les pieds, font des pas plus facilement et donc la marche s'améliore. La longueur de foulée augmente. Le patient signale qu'il se sent plus en équilibre et en sécurité. Il devient plus facile et moins dangereux de descendre les escaliers et de descendre du trottoir. Dans certains cas, le recours à un bâton de marche est réduit.

Cependant, des problèmes ont été signalés. Comme le montre la figure 17, même si la tête est relevée, la posture du dos change, ce qui crée un inconfort initial dans les muscles des jambes et dans le bas du dos. Cela peut s'améliorer avec le temps à mesure que les muscles se renforcent. L'adaptation à la nouvelle posture dépend beaucoup d'autres facteurs, comme les problèmes sous-jacents au dos et aux genoux. S'il n'y a pas de problèmes orthopédiques majeurs, le patient signale une adaptation rapide au nouvel arrangement et une amélioration générale de la mobilité et du sentiment de sécurité.

Dans quelques cas étudiés, après plusieurs mois, les lunettes ont été retirées pour voir si le renforcement des muscles du corps était permanent. La posture est revenue à sa position d'origine et le patient a ressenti la même insécurité qu'auparavant pendant le mouvement.

PROBLÈMES LIÉS À LA PRESCRIPTION DE PRISMES POUR ÉQUILIBRER LA POSTURE

Il est important de noter qu'aucune correction prismatique de la posture ne devrait être intégrée à la prescription si elle compromet les besoins visuels ou l'équilibre musculaire binoculaire du patient.

Les recherches menées par Huang et Ciuffreda suggèrent qu'il y a une adaptation sensorimotrice et perceptuelle rapide, évaluée objectivement et subjectivement, en réponse à des prismes jumelés verticaux ${ }^{33}$. Les prismes de plus de $8^{\Delta}$ peuvent entraîner une réduction de la qualité de la vision et doivent être évités. La perception à travers un prisme provoque l'expansion de l'image lorsque le sujet regarde vers la base, et sa compression lorsqu'il regarde vers le haut. Erismann et Köhler ont suggéré que les patients s'adaptent à cet effet $^{34}$. De plus, une ligne horizontale observée au-dessus du niveau des yeux semble inclinée dans la direction de la base, tandis qu'une ligne au-dessous du niveau des yeux tend à s'incliner vers le haut. Des patients ont signalé la courbure des lignes droites dans les prescriptions de verres plano avec des prismes de $4 \Delta^{35,36}$ Les patients qui ont des ordonnances élevées ressentent ces effets avec les lentilles unifocales lorsqu'ils regardent à l'extérieur du centre optique, mais en compensant avec l'ajustement de la tête, la vision peut être réalignée avec le centre optique. Cet ajustement n'est pas possible lorsque les prismes jumelés sont inclus dans l'ordonnance. Dans leur étude sur l'adaptation des patients aux prismes jumelés, Sheedy et Parsons ${ }^{2}$ ont noté que les primes jumelés de $2^{\Delta}$ ou moins étaient facilement tolérés, tandis que ceux 
de $4^{\Delta}$ prismes étaient rejetés. D’après mon expérience, les prismes jumelés verticaux de $3^{\Delta}$ constituent la limite. Dans des cas exceptionnels, où un prisme plus élevé était nécessaire, il s'est avéré efficace. Dans le cas des prismes jumelés horizontaux, 5-6 étaient tolérées.

DÉTERMINER SI DES PRISMES DOIVENT ÊTRE PRESCRITS

La réaction des patients qui reçoivent des prismes jumelés est idiosyncrasique. Pour tenter de réduire la nontolérance, on utilise une autre étape au moment de déterminer si des prismes jumelés conviennent. Ce test est totalement subjectif et le résultat ne peut pas être facilement vérifié par des mesures objectives. On demande au patient de dire s'il a l'impression que sa posture est droite, ou s'il se penche vers l'avant ou vers l'arrière. Comme il faut aussi tenir compte de la stabilité du patient, il est important que la puissance du prisme soit ajustée jusqu'à ce que le patient signale qu'il se sent en équilibre.

Quaid a suggéré qu'en observant la marche du patient, le praticien peut avoir une idée de la pertinence de prescrire des prismes (correspondance personnelle).

On demande au patient de traverser une pièce en marchant, et les changements nécessaires sont apportés à la prescription prismatique selon le sentiment de sécurité et de confort. Dans le cas d'une personne âgée, on demande au patient de se tenir debout et de marcher, sans utiliser d'aide comme un bâton de marche. Si le patient utilise un déambulateur (cadre Zimmer), l'essai est effectué avec le déambulateur.

Il existe des techniques pour déterminer si les prescriptions de prismes modifient l'équilibre corporel. Ces techniques sont utilisées principalement pour prescrire des prismes horizontaux. Le sujet marche le long d'un tapis avec capteurs de pression, et on comparaison les pressions différentielles exercées par chaque pied alors qu'il marche ${ }^{3}$. Cela ne convient pas aux prismes verticaux.

Les prismes jumelés changent la sensation de hauteur, et le patient peut avoir besoin de quelques jours d'ajustement. Les prescriptions élevées, négatives ou positives, sont souvent montées sur l'axe horizontal de la monture, sans que les centres optiques soient bien situés. Ceci introduit des prismes jumelés. Il est important d'en être conscient au moment de prescrire et de fabriquer des lunettes.

\section{CONCLUSION}

Bien que le traitement des anomalies orthopédiques par l'adaptation visuelle ait déjà été mis au point par des chercheurs, l'intégration de ces méthodes dans la pratique optométrique de routine a souvent été mise de côté. Il est rare qu'on insiste sur ces méthodes dans la plupart des programmes de formation en optométrie. Étant donné que le champ d'exercice de la profession optométrique change en raison des pressions économiques, le traitement des troubles orthopédiques par les méthodes optométriques devrait être intégré à la pratique courante.

\section{REMERCIEMENTS}

Je tiens à remercier Dr Patrick Quaid de son immense aide pour clarifier bon nombre des concepts présentés ici, ainsi que du temps et des efforts qu'il a consacrés à la préparation de ce document.

Les patients présentés dans cet article sont ceux que je reçois à mon cabinet et ont contribué de leur temps à mes projets. Ils ont tous donné leur permission pour que leurs dossiers soient publiés. Je les remercie de leur aide.

Enfin, j'aimerais remercier mon épouse, Ruthie, du temps qu'elle a consacré à la révision de ce document.

CONTACT

Courriel : shapiro.yonatan@gmail.com

Adresse postale : POB 1282, Pardes Hanna, Israël, 37000 


\section{RÉFÉRENCES}

1. Kaplan M. Vertical yoked prisms. Optometric Extension Program Continuing Education Courses. Santa Ana, CA: Optometric Extension Program Foundation, 1978-1979.

2. Sheedy JE, Parsons SD. Vertical yoked prisms-patient acceptance and postural adjustment. Ophthal Physiol Opt 1987;7(3):255-7. doi. org/10.1111/j.1475-1313.1987.tb00742.

3. Padula WV, Subramanian P, Spurling A, Jennes J. Risk of fall (RoF) intervention by affecting visual egocenter through gait analysis and yoked prisms. NeuroRehabilitation 2015;37(2):305-14. doi: 10.3233/ NRE-151263.

4. Eubank T, Cool T. Improving guided action and perception through use of prisms. J Am Optom Assoc 2001;72(4):217-26.

5. Schmid KL, Beavis SD, Wallace SI, et al. The effect of vertically yoked prisms on binocular vision and accommodation. Optom Vis Sci 2019;96(6):414-23. doi: 10.1097/OPX.0000000000001388

6. Asper L, Leung A, Tran C, Suttle CM, Watt K. The effects of vertical yoked prism on horizontal heterophoria. Optom Vis Sci 2015;92(10):1016-20. doi:10.1097/OPX.0000000000000686.

7. Bansal S, Han E, Ciuffreda KJ. Use of yoked prisms in patients with acquired brain injury: A retrospective analysis. Brain Inj 2014;28(11):1441-6. doi.org/10.3109/02699052.2014.919527

8. Zasler N, Katz D, Ross M. Brain injury medicine: principles and practice. New York, NY: Demos Medical, 2007:523

9. Ashley MJ. Traumatic brain injury, rehabilitation, treatment and case management. Boca Raton, FL: CRC Press, 2010.

10. Suter P, Harvey L. Vision rehabilitation. Boca Raton, FL: CRC Press, 201:209.

11. Padula WV, Argyris S, Ray J. Visual evoked potentials evaluating treatment for post-trauma visions syndrome in patients with traumatic brain injuries. Brain Inj 1994;8(2):125-33.

12. Padula WV, Munitz R, Magrun M, eds. Neuro-visual processing: An integrated model of rehabilitation. Santa Ana, CA: Optometric Extension Program Press, 2012.

13. Padula WV, Nelson CA, Benabib R, Yilmaz C, Krevisky S. Modifying postural adaptation following a CVA through prismatic shift of visuo-spatial egocenter. Brain Inj 2009;23(6):566-76.

14. Velay JL, Roll R, Lennerstrand G, Roll JP. Eye proprioception and visual localization in humans: Influence of ocular dominance and visual context. Vis Res 1994;34(16):2169-76.

15. Rotating tunnel optical illusion. YouTube Web site. https://www. youtube.com/watch?v=hUW6516VJA4. Published May 13, 2017. Accessed November 16, 2019.

16. Quaid P, Hamilton-Wright A. Diagnosing extraocular muscle dysfunction in clinic: comparing computerized Hess Analysis, Park's 3-Step Test and a novel 3-Step Test. Optom Vis Dev 2010;41(3):143-57.

17. Urrets-Zavalía A Jr, Solares-Zamora J, Olmos HR. Anthropological studies on the nature of cyclovertical squint. Br J Ophthalmol 1961 Sep;45(9):578-96.

18. Urrets-Zavalía A Jr. Significance of congenital cyclo-vertical motor defects of the eyes. Br J Ophthalmol 1955 Jan;39(1):11-20.
19. Shapiro IJ. Parallel-testing infinity balance. Instrument and technique for the parallel testing of binocular vision. Optom Vis Sci 1995;72(12):916-23.

20. Shapiro IJ. A new instrument and technique of refraction and binocular balance. The Optician (UK) 1998;215(5643):34-40.

21. Quaid P, Simpson T. Association between reading speed, cycloplegic refractive error, and oculomotor function in reading disabled children versus controls. Graefes Arch Clin Exp Ophthalmol 2013;251:169-87. DOI 10.1007/s00417-012-2135-0

22. Shapiro IJ. Relation between vertical facial asymmetry and postural changes of the spine and ancillary muscles. Optom Vis Sci 1994;71(8):529-38.

23. Shapiro IJ. Examination techniques in cases of vertical facial asymmetry. The Optician (UK) 1993;205(5402):20-7.

24. Major A, Maples WC, Toomey S, DeRosier W, Gahn D. Variables associated with the incidence of infantile esotropia. Optometry 2007;78:534-41

25. Aygit ED, Kocamaz M, Inal A, et al. Management of Duane retraction syndrome with prismatic glasses. Clin Ophthalmol 2017;11:697-700.

26. Shapiro IJ. The use of Yoked prisms to improve the appearance and function of slack-jaw in cases of hypotonia. Optometry Today (UK) 2006;12:38-9.

27. Goldberg EM, McCreedy EM, Gettel CJ, Merchant RC. Slipping through the cracks: A cross-sectional study examining older adult emergency department patient fall history, post-fall treatment and prevention. R I Med J 2017;100(12):18-23.

28. Gelbard R, Inaba K, Okoye OT, et al. Falls in the elderly: a modern look at an old problem. Am J Surg 2014;208(2):249-53.

29. Elliot D. Blurred vision, spectacle correction and falls in older adults. Optom Vis Sci 2014;91(6):593-601.

30. Adams T. Connecting falls to elder vision. Optom Vis Sci 2014;91(6):591-2.

31. Shapiro IJ. The effect of vertical facial asymmetry on the fitting of progressive addition lenses (PAL). Optometry Update (Israel) 1994;1(2):13-5.

32. Haran MJ, Cameron ID, Ivers RQ, et al. Effect on falls of providing single vision distance glasses to multifocal glasses wearers: visible randomised controlled trial. BMJ 2010:340:c2265.

33. Huang MA, Ciuffreda KJ. Short-term adaptation to vertical yoked prisms. Optom Vis Sci 2006;83(4):242-8.

34. Sachse P, Beermann U, Martini M, Maran T, Domeier M, Furtner MR. "The world is upside down" The Innsbruck Goggle Experiments of Theodor Erismann (1883-1961) and Ivo Kohler (1915-1985). Cortex 2017;92:222-32. doi: 10.1016/j.cortex.2017.04.014.

35. Atchison DA, Suheimat M. Theoretical study of refraction effects of plano ophthalmic prisms. Optom Vis Sci 2019;96(1):35-42. doi: 10.1097/OPX.0000000000001321.

36. Atchison DA, Lu J, Yip C, Suheimat M, Schmid KL. Experimental study of refraction effects of nominally plano ophthalmic prisms and magnifying lenses. Optom Vis Sci 2019;96(2):111-6. doi: 10.1097/ OPX.0000000000001334. 